\title{
Fourier 变换成像光谱技术
}

\author{
相里斌，吕群波，才啟胜，方煜，周锦松，黄昱 \\ 中国科学院计算光学成像技术重点实验室, 空天信息创新研究院, 北京 100094 \\ * 通信作者. E-mail: xiangli@cashq.ac.cn \\ 收稿日期: 2020-05-29; 接受日期: 2020-06-15; 网络出版日期: 2020-10-15
}

\begin{abstract}
摘要 成像光谱仪能够获取目标的几何和光谱辐射信息, 是一种具有量化分析和特征识别能力的光 学感知手段, 按照分光方式可分为棱镜色散、光栅衍射和干涉调制等多种类型. Fourier 变换成像光谱 技术通常基于双光束干涉原理, 将探测器获取的干涉图通过 Fourier 变换反演出目标光谱. 该技术具 有高通量、多通道、高光谱分辨率等优点, 是成像光谱技术中最具创新空间和应用潜力的重要研究方 向, 发展过程中逐步形成了时间调制、空间调制、时空调制等多种类型, 在工业农业、科学研究、生物 医药、大气探测、环境监测、资源调查等诸多领域得到广泛应用。本文从 Michelson 千涉仪原理出发, 简要介绍 Fourier 变换光谱技术的基本理论, 按照 3 种干涉调制类型分别介绍不同 Fourier 变换成像 光谱技术的典型方案、突出特点和主要应用。
\end{abstract}

关键词 Fourier 变换, 成像光谱, 辐通量, 多通道, 调制度, 并行采样

\section{1 引言}

1665 年, Newton 利用三棱镜将太阳光色散成彩色光带, 首次意识到光谱的存在 ${ }^{[1]} .1859$ 年, Kirchhoff 和 Bunsen ${ }^{[2]}$ 研制了世界上第 1 台光谱仪, 并对不同物质产生的光谱开展研究, 创建了光谱分析 法. 早期的光谱仪通常采用棱镜或光栅作为分光元件, 1880 年, Michelson ${ }^{[3]}$ 发明了以他名字命名的干 涉仪, 1887 年, Michelson 和 Morley ${ }^{[4]}$ 利用干涉仪精确测量两垂直光束的速度, 揭示了光速不变性, 证 明以太不存在, 为狭义相对论的诞生提供了实验依据. 1892 年, Rayleigh ${ }^{[5]}$ 发现双光束干涉强度与光 源光谱间存在 Fourier 变换关系, 由于当时缺乏高效的计算工具用于光谱反演, 该技术一直未得到推 广应用. 1965 年, 随着计算机技术的发展和快速 Fourier 变换算法的出现 ${ }^{[6]}$, 基于 Michelson 干涉仪等 的光谱测量技术迅速兴起, 称为 Fourier 变换光谱技术 (Fourier transform spectroscopy, FTS).

Fourier 变换光谱仪可以实现极高的光谱分辨率, 还具有高通量优点 ${ }^{[7]}$ 和多通道优点 ${ }^{[8]}$, 这使其 拥有了高光谱分辨率、高灵敏度和高信噪比等性能优势. 至今, FTS 已发展成为先进科学仪器的典型 代表, 在工业、农业、科研、卫生、环境、资源等诸多领域得到了非常广泛的应用.

\footnotetext{
引用格式: 相里斌, 吕群波, 才啟胜, 等. Fourier 变换成像光谱技术. 中国科学: 信息科学, 2020, 50: 1462-1474, doi: 10.1360/ SSI-2020-0150

Xiangli B, Lv Q B, Cai Q S, et al. Fourier transform imaging spectroscopy (in Chinese). Sci Sin Inform, 2020, 50: 1462-1474, doi: 10.1360/SSI-2020-0150
} 


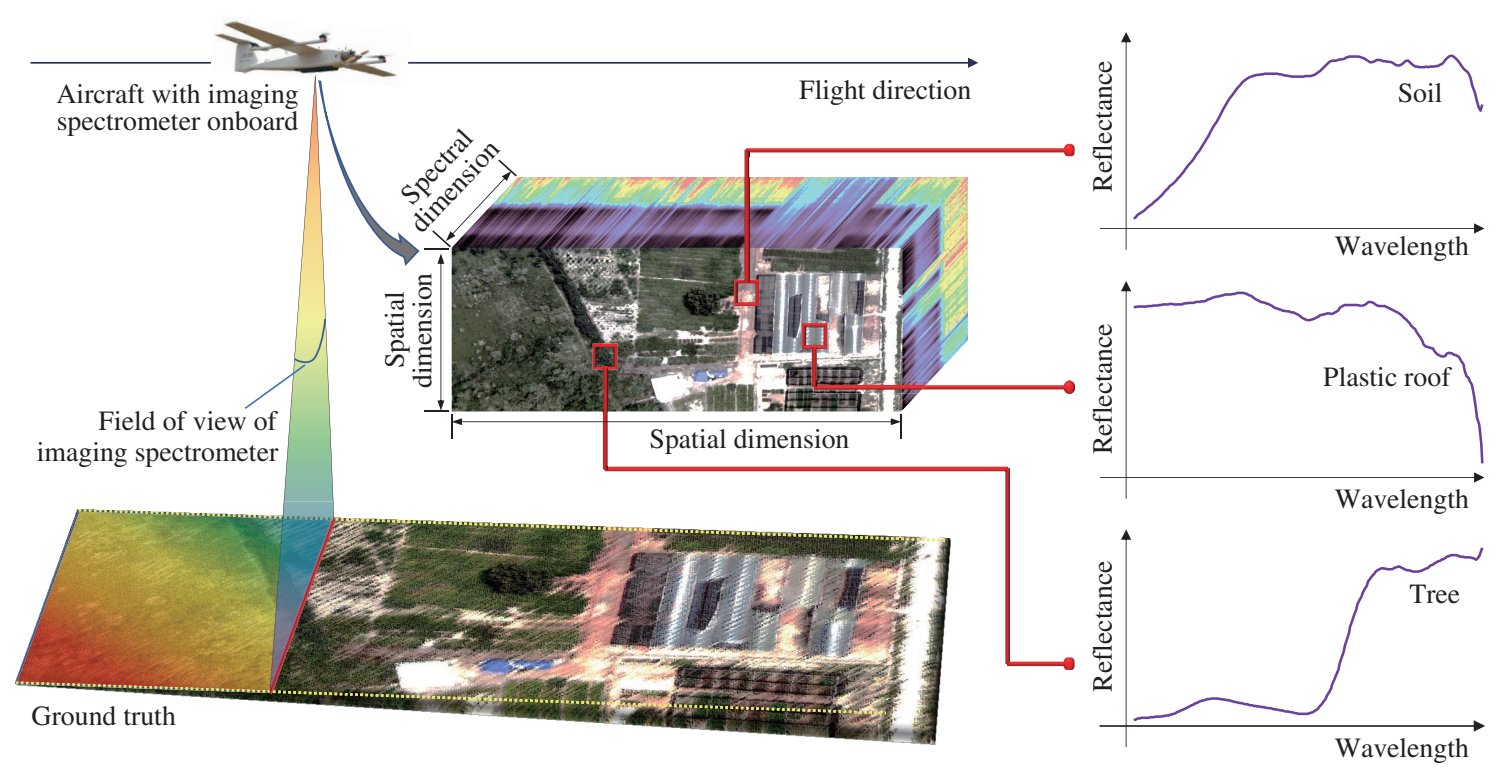

图 1 (网络版彩图) 成像光谱技术概念示意图

Figure 1 (Color online) Schematic diagram of imaging spectroscopy

20 世纪 80 年代初, 随着航空航天技术的发展和光电探测器性能的突破, 成像光谱技术 (imaging spectroscopy) 作为一种具备多元信息获取能力的新型光学遥感手段应运而生 [9]. 成像光谱仪既可以 获得目标的几何影像, 又可以获得对应光谱辐射信息, 该图谱数据被称作数据立方体 (datacube), 如图 1 所示. 成像光谱仪的分光方式主要有滤波、色散、衍射和干涉等, 其中, Fourier 变换成像光谱技术 (Fourier transform imaging spectroscopy, FTIS) 采用干涉分光方式, 继承了 FTS 的主要优点, 发展形 成了时间调制、空间调制和时空调制等多种类型, 在高光谱分辨率、高空间分辨率和高信噪比图谱信 息获取方面具有广阔的创新空间和巨大的应用潜力.

\section{Fourier 变换光谱技术原理}

Fourier 变换光谱仪的核心是干涉仪, Michelson 干涉仪是诸多形式干涉仪的经典. 图 2 给出基于 Michelson 干涉仪的 FTS 原理示意图. 光源发出的光经准直镜后, 通过分束器分为两束相干光, 一束 光传输到固定反射镜, 再被反射回分束器, 部分透射到会聚镜至探测器; 另一束光传输到运动反射镜, 再被反射回分束器, 部分反射到会聚镜至探测器. 两束会聚光在探测器处发生干涉. 随着动镜的移动, 干涉信号的相位差不断变化, 探测器接收到的干涉信号形成一条曲线, 称作干涉图 (interferogram).

记光源发出的单色光波数为 $\nu$ (波长 $\lambda$ 的倒数), 振幅为 $a$, 分束器的反射率为 $r$, 透射率为 $t$, 则干 涉图可以表达为

$$
I(x, \nu)=\{r t a[1+\exp (-i \phi)]\} \times\{r t a[1+\exp (-i \phi)]\}^{*} .
$$

令 $R=r \times r^{*}, T=t \times t^{*}, B(\nu)=a \times a^{*}$, 式 (1) 可以简化为

$$
I(x, \nu)=2 R T B(\nu)(1+\cos \phi),
$$

其中, $B(\nu)$ 为光源的光谱辐亮度, $\phi=2 \pi \nu x$ 为动镜与定镜间的相位差, $x$ 为光程差. 


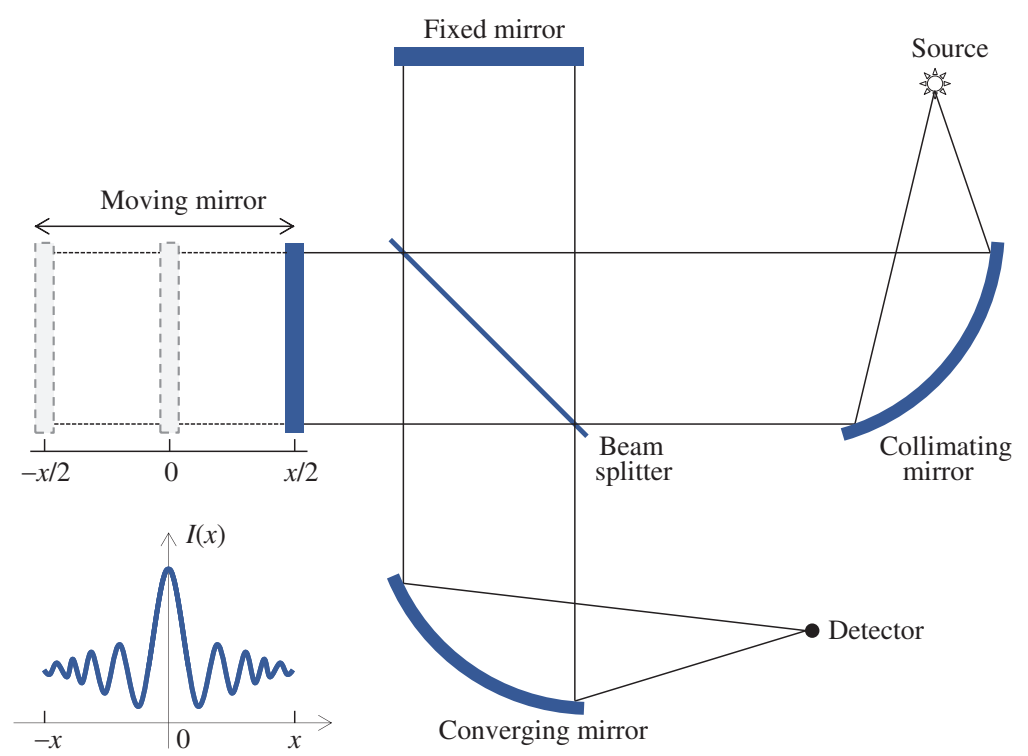

图 2 (网络版彩图) 基于 Michelson 干涉仪的 FTS 原理图

Figure 2 (Color online) Principle of FTS with Michelson interferometer

全谱段干涉图是各单色光干涉图之和 (以下忽略常数项):

$$
I(x)=\int_{0}^{\infty} I(x, \nu) \mathrm{d} \nu=\int_{0}^{\infty} B(\nu) \cos (2 \pi \nu x) \mathrm{d} \nu .
$$

现实中 $B(\nu)$ 为实函数, $I(x)$ 为偶函数, 将干涉图偶延拓得到

$$
I(x)=\int_{-\infty}^{\infty} B(\nu) \exp (-i 2 \pi \nu x) \mathrm{d} \nu=F\{B(\nu)\},
$$

即干涉图与入射光谱存在 Fourier 变换关系. 因此, 理论上只需对干涉图进行逆 Fourier 变换即可复原 出光源光谱.

对于实际 FTS 系统, 光谱范围和光程差都是有限的, 干涉图和复原光谱分别为

$$
\begin{gathered}
I(x)=\int_{\nu_{1}}^{\nu_{2}} B(\nu) \exp (-i 2 \pi \nu x) \mathrm{d} \nu=\int_{-\infty}^{\infty} T_{B}(\nu) B(\nu) \exp (-i 2 \pi \nu x) \mathrm{d} \nu, \\
B^{\prime}(\nu)=\int_{-L}^{L} I(x) \exp (i 2 \pi \nu x) \mathrm{d} x=\int_{-\infty}^{\infty} T_{I}(x) I(x) \exp (i 2 \pi \nu x) \mathrm{d} x,
\end{gathered}
$$

其中, $\nu_{1}, \nu_{2}$ 为波数范围, $L$ 是最大光程差, 决定了仪器可分辨波数的半高宽:

$$
\delta \nu=\frac{1}{2 L} .
$$

仪器的光谱分辨率为

$$
R=\frac{\lambda}{\delta \lambda}=\frac{\nu}{\delta \nu} .
$$

$T_{I}$ 是截断函数, 其 Fourier 变换为仪器线型函数 (instrument line shape, ILS). Fourier 变换的性质 决定了仪器线型函数存在 Gibbs 现象, 通常需采用切趾函数 (apodization function) 来抑制 Gibbs 振荡 
的旁瓣, 包括三角函数、Gaussian 函数、Happ-Genzel 函数、Hamming 函数等 ${ }^{[10]}$. 切趾函数在抑制旁 瓣的同时会降低光谱分辨率.

干涉图采样过程中还存在相位误差, 在 Fourier 变换复原光谱之前需要对干涉图进行相位修正, 主 要有平方根法、Mertz 法 [11] 和 Forman 法 [12] 等.

此外, 实际系统中不可避免地存在噪声, 系统噪声包括诸多因素 ${ }^{[13]}$ :

$$
\sigma_{\text {system }}=\sqrt{\sigma_{\text {photon }}^{2}+\sigma_{\text {pattern }}^{2}+\sigma_{\text {reset }}^{2}+\sigma_{\text {read }}^{2}+\sigma_{\text {dark }}^{2}+\sigma_{\text {ADC }}^{2}},
$$

其中, $\sigma_{\text {photon }}$ 为光子噪声, $\sigma_{\text {pattern }}$ 为模式噪声, $\sigma_{\text {reset }}$ 为复位噪声, $\sigma_{\text {read }}$ 为读出噪声, $\sigma_{\text {dark }}$ 为暗电流, $\sigma_{\mathrm{ADC}}$ 为量化噪声.

当光子噪声远大于其他噪声时, 称光子噪声受限, 此时干涉图的信噪比 ${ }^{[14]}$ :

$$
\begin{aligned}
\operatorname{SNR}_{I} & =\frac{N_{p}(x)}{\sigma_{\text {system }}} \approx \frac{N_{p}(x)}{\sigma_{s}}=\sqrt{N_{p}(x)} \\
& =\sqrt{\frac{\pi}{4} \int_{\nu_{1}}^{\nu_{2}} \frac{B(\nu) \cdot s^{2} \cdot \Delta t \cdot \tau(\nu)}{h \cdot c \cdot \nu \cdot(F \#)^{2}}[1+\cos (2 \pi \nu x)] \mathrm{d} \nu}
\end{aligned}
$$

其中, $N_{p}(x)$ 是干涉图对应的光子数, $s$ 为正方形探测器像元边长, $\Delta t$ 为探测器积分时间, $F \#$ 为会聚 镜的 $F$ 数, $\tau(\nu)$ 为光学系统的透过率, $h$ 为 Planck 常数, $c$ 为真空光速.

当系统噪声以探测器读出噪声、模式噪声和暗电流噪声等为主时, 称探测器噪声受限, 干涉图的 信噪比:

$$
\begin{aligned}
\mathrm{SNR}_{I} & =\frac{N_{e}(x)}{\sigma_{\text {system }}} \approx \frac{N_{e}(x)}{\sqrt{\sigma_{\text {pattern }}^{2}+\sigma_{\text {read }}^{2}+\sigma_{\text {dark }}^{2}}} \\
& =\frac{\frac{\pi}{4} \int_{\nu_{1}}^{\nu_{2}} \frac{B(\nu) \cdot s^{2} \cdot \Delta t \cdot \tau(\nu)}{h \cdot \cdot \cdot \cdot \cdot(F \#)^{2}}[1+\cos (2 \pi \nu x)] \mathrm{d} \nu}{\sqrt{\sigma_{\text {pattern }}^{2}+\sigma_{\text {read }}^{2}+\sigma_{\text {dark }}^{2}}},
\end{aligned}
$$

其中 $N_{e}(x)$ 为干涉图对应的电子数. 在探测器噪声受限条件下, Fourier 变换光谱仪的多通道优势明 显, 具有较高的信噪比.

\section{3 时间调制 Fourier 变换成像光谱技术}

时间调制 Fourier 变换成像光谱技术 (temporally modulated fourier transform imaging spectroscopy, TMFTIS) 原理与图 2 类似, 通过适当的光学设计和采用面阵探测器等, 具备了测量不同视场光源光谱 的能力, 即成像能力. 由于光程差在动镜运动过程中随时间变化, 干涉图可表达为时间的函数, 故称时 间调制。

采用平面动镜 Michelson 干涉仪的时间调制 Fourier 变换成像光谱仪, 动镜运动过程中发生倾斜, 会导致干涉图调制度降低, 影响复原光谱质量. 如图 3 所示, 仅以零视场光束进行分析, 记倾斜中心 $O$ 距零光程差位置 $x_{0}$, 动镜倾斜角度 $\beta$, 入射光束口径为 $D$, 入射光束中心光线与 $O$ 的距离为 $\varepsilon$.

考虑单色光情形, 设入射光束截面为矩形, 可以得到干涉图:

$$
I\left(x_{0}\right)=\int_{-(D / 2-\varepsilon)}^{D / 2+\varepsilon} B(\nu) \cos \left[2 \pi \nu\left(x_{0}+2 \xi \beta\right)\right] \mathrm{d} \xi=B(\nu) \operatorname{sinc}(2 \pi \nu D \beta) \cos \left[2 \pi \nu\left(x_{0}+2 \varepsilon \beta\right)\right] .
$$




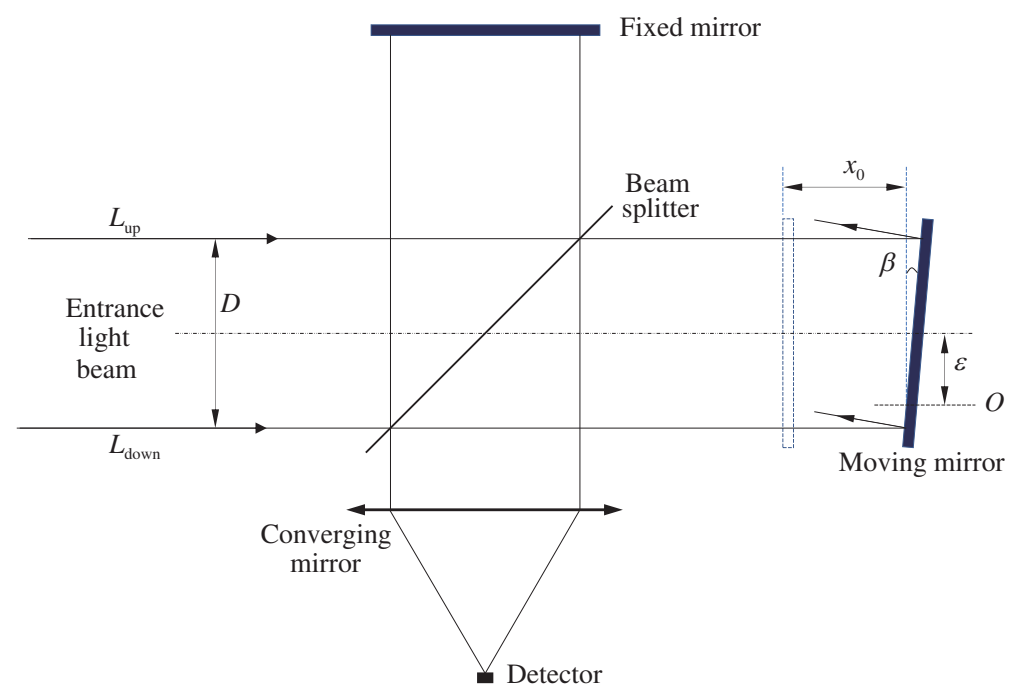

图 3 (网络版彩图) Michelson 干涉仪动镜倾斜示意图

Figure 3 (Color online) Moving mirror tilted in Michelson interferometer

可见, 当平面动镜倾斜时, 干涉图被一个 sinc 函数调制, 记调制度为

$$
M(D, \beta)=\operatorname{sinc}(2 \pi \nu D \beta) .
$$

如果要求平面动镜倾斜导致的干涉图调制度下降不超过 $10 \%$, 即

$$
M\left(D, \beta_{\max }\right)=\operatorname{sinc}\left(2 \pi \nu D \beta_{\max }\right)=0.9,
$$

得到平面动镜容许的最大倾角为

$$
\beta_{\max }=\frac{\lambda}{8 D} .
$$

取 $\lambda=632.8 \mathrm{~nm}, D=50 \mathrm{~mm}$, 得 $\beta=0.33^{\prime \prime}$.

可见, 时间调制 Fourier 变换成像光谱仪对平面动镜倾斜的要求非常严格, 因此, 高平稳性的动镜 机构是必须要解决的关键技术. 通常采用动镜倾斜实时测量与压电晶体高速驱动静镜补偿的方法来克 服平面动镜倾斜误差的影响 ${ }^{[15]}$, 同时, 利用立方角镜或者猫眼镜出射光束与入射光束反向平行、双平 行平面镜出射光束与入射光束同向平行的特点, 可设计对运动误差不敏感的动镜系统. 图 4 给出了双 角镜摆臂干涉仪 [16]、角反射转镜干涉仪 [17]、平行镜摆动干涉仪 ${ }^{[18]}$ 方案示意图, 这些方案的要点是 在反射镜运动过程中, 即使发生倾斜也不影响出射相干光束的平行度, 进而保证了干涉图的调制度.

时间调制 Fourier 变换成像光谱仪具有辐通量 (throughput) 高的优点, 同时, 动镜运动范围很大, 能 够实现极高光谱分辨率, 应用十分广泛. 在遥感领域主要以大气成份分析、痕量气体探测为主. 1996 年 日本发射的先进地球观测卫星 (ADEOS) 搭载的温室气体监测干涉仪 [19]、2016 年中国发射的 “风云 四号” 卫星搭载的干涉式大气垂直探测仪 ${ }^{[20]}$, 采用了平面动镜方案; 2003 年加拿大发射的大气化学实 验卫星 (ACE) 搭载的 Fourier 变换光谱仪 ${ }^{[21]} 、 2009$ 年日本发射的温室气体观测卫星 (GOSAT) 搭载 的热红外和近红外碳观测 Fourier 变换光谱仪 [22]、2018 年中国 “高分五号” 卫星搭载了大气环境红外 甚高光谱分辨率探测仪 ${ }^{[23]}$, 采用了双角镜摆臂干涉仪方案. 


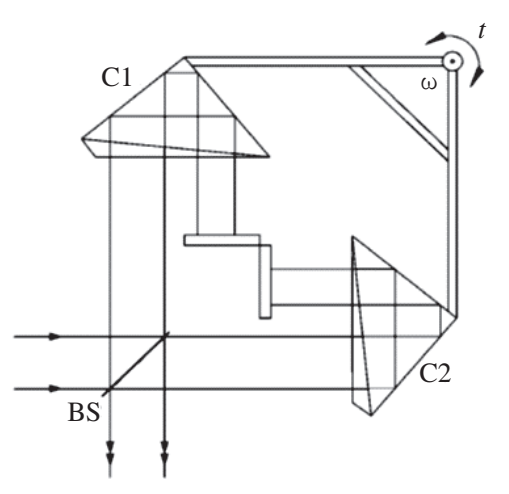

(a)

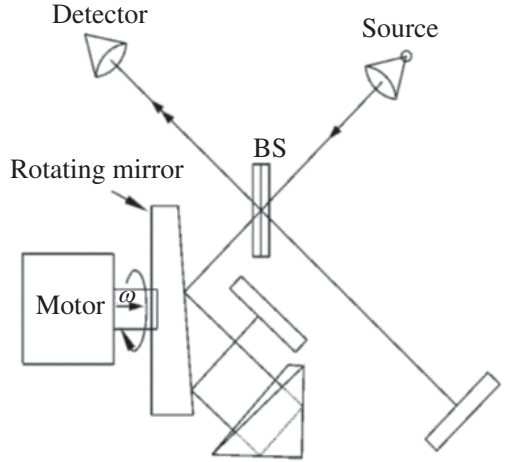

(b)

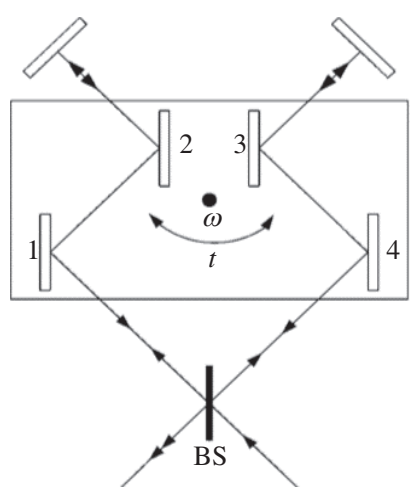

(c)

图 43 种类型高稳定度 TMFTIS

Figure 4 Three TMFTIS types of high stability. (a) TMFTIS based on double pendulum interferometer; (b) TMFTIS based on ultra-rapid-scanning interferometer; (c) TMFTIS based on Perkin-Elmer Dynascan interferometer
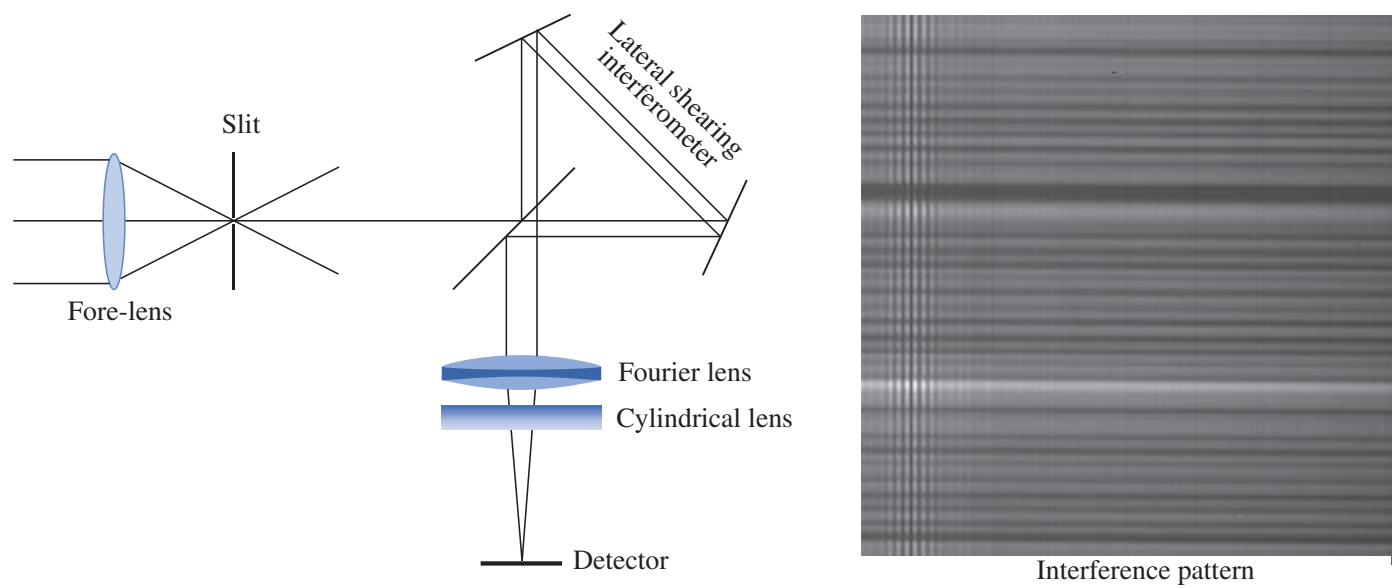

图 5 (网络版彩图) SMFTIS 原理示意图及干涉图案

Figure 5 (Color online) Principle of SMFTIS and the interference pattern

\section{4 空间调制 Fourier 变换成像光谱技术}

随着 Fourier 变换成像光谱技术应用领域的不断拓展, 20 世纪 90 年代, 出现了静态 Fourier 变换 成像光谱技术, 其主要特点是引入狭缝、没有动镜, 采用横向剪切干涉仪将狭缝剪切相干, 干涉图案按 照不同相位差分布在面阵探测器的两维空间上, 称为空间调制 Fourier 变换成像光谱技术 ${ }^{[24]}$ (spatially modulated Fourier transform imaging spectroscopy, SMFTIS).

空间调制 Fourier 变换成像光谱技术的基本原理如图 5 所示, 由前置镜、入射狭缝、Sagnac 横向 剪切干涉仪、Fourier 镜、柱面镜、探测器等部分组成. 狭缝方向与柱面镜母线垂直, 位于前置镜的后 焦面同时也是 Fourier 镜的前焦面上, 横向剪切干涉仪将狭缝剪切为两个相干虚狭缝, 经 Fourier 镜后 发生干涉, 柱面镜将干涉图案一维聚焦到探测器.

设两个虚狭缝在垂直光轴方向的剪切量为 $\zeta$, 自虚狭缝发出与光轴夹角 $\theta$ 的相干光束经 Fourier 


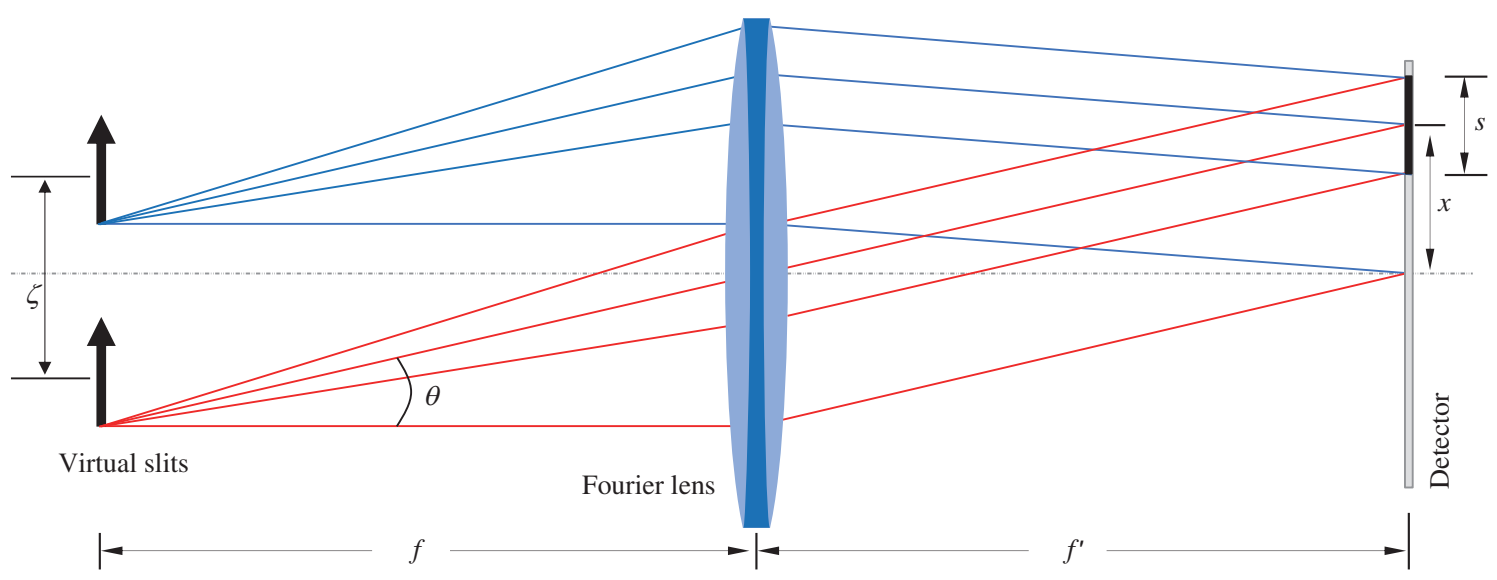

图 6 (网络版彩图) SMFTIS 光学等效模型

Figure 6 (Color online) Optical equivalent model of SMFTIS

镜和柱面镜后, 在探测器距离零光程差 $x$ 处发生干涉, 此位置光程差为

$$
\delta=\zeta \cdot \sin \theta=\frac{\zeta \cdot x}{f^{\prime}},
$$

其中 $f^{\prime}$ 为 Fourier 镜像方焦距. 则该点对应的干涉图为

$$
I(x)=\int_{0}^{\infty} B(\nu) \cos \left(2 \pi \nu \frac{\zeta x}{f^{\prime}}\right) \mathrm{d} \nu .
$$

空间调制 Fourier 变换成像光谱仪单次曝光可获得狭缝对应的一列目标点的干涉图, 处理后得到 相应的复原光谱. 当仪器沿垂直狭缝方向逐列扫描, 就可以得到另一维空间信息和相应的光谱信息, 形 成数据立方体.

空间调制 Fourier 变换成像光谱仪采用面阵探测器采集干涉图, 由于像元尺寸的影响, 干涉图的 调制度会有所降低. 图 6 为简化的光学等效模型.

对于波数为 $\nu$ 的单色光, 设探测器填充因子 $100 \%$, 像元边长 $s$, 则干涉图为 ${ }^{[25]}$

$$
I(x)=\int_{x-s / 2}^{x+s / 2} B(\nu) \cdot \exp \left(-i 2 \pi \nu \frac{\zeta x}{f^{\prime}}\right) \mathrm{d} x=B(\nu) \cdot \operatorname{sinc}\left(\frac{\zeta s \nu}{f^{\prime}}\right) \cdot \exp \left(-i 2 \pi \nu \frac{\zeta x}{f^{\prime}}\right),
$$

即干涉图调制度为

$$
M_{I}(\nu)=\operatorname{sinc}\left(\frac{\zeta s \nu}{f^{\prime}}\right),
$$

其中, $\zeta s / f^{\prime}$ 是干涉图的采样间隔. 根据 Nyquist 采样定理, 须满足

$$
\frac{\zeta s}{f^{\prime}} \leqslant \frac{1}{2 \nu} \text {. }
$$

此时干涉图调制度

$$
M_{I}(\nu) \geqslant \operatorname{sinc}\left(\frac{1}{2}\right)
$$

这是一个具有应用意义的量化判据. 
表 1 不同横向剪切干涉仪的干涉图调制度表达式

Table 1 Interference modulation depth of different lateral shearing interferometers

\begin{tabular}{cc}
\hline Lateral shearing interferometer & Modulation depth \\
\hline Sagnac & $M_{\mathrm{I}}(\nu)=1$ \\
Mach-Zehnder & $M_{\mathrm{I}}(\nu)=1$ \\
Lloyd & $M_{\mathrm{I}}(\nu)=\operatorname{sinc}(2 \nu w \sin \theta)$ \\
Fresnel & $M_{\mathrm{I}}(\nu)=\operatorname{sinc}(2 \nu w \sin \alpha \cos \theta)$ \\
\hline
\end{tabular}

从式 (17) 可知, Sagnac 横向剪切干涉仪本身没有导致干涉图调制度的下降. 但是, 除 Sagnac 横 向剪切干涉仪外, 还有多种横向剪切干涉仪可用于空间调制 Fourier 变换成像光谱技术, 包括 MachZehnder 干涉仪、Lloyd 镜、Fresnel 镜、双折射棱镜等, 不同横向剪切干涉仪对干涉图调制度的影响也 不同, 如表 1 所示. 干涉图调制度分析为优化设计 Fourier 变换成像光谱仪提供了一种客观判据, 由于 干涉图调制度最终体现在对复原光谱的影响, 该方法也称光谱传递函数分析 ${ }^{[26]}$.

当横向剪切干涉仪不影响干涉图调制度时, 空间调制 Fourier 变换成像光谱仪的狭缝宽度只取决 于空间分辨率, 与光谱分辨率无关, 这是相对于光栅或棱镜分光方式的一个优点, 意味着如果在狭缝宽 度方向上空间分辨率不高, 系统能够拥有较高的辐通量. 但在多数应用情况下狭缝是很窄的, 这使空 间调制技术常常不再具备高通量优势.

空间调制 Fourier 变换成像光谱技术为航天高光谱成像遥感探索了一个新途径, 其结构紧凑, 性能 稳定, 兼顾了空间分辨率和光谱分辨率, 在资源、环境遥感领域得到应用. 2000 年, 美国发射了 “MightySat II" 卫星, 搭载了国际上首台空间调制 Fourier 变换成像光谱仪开展对地遥感和地球临边探测技术 试验 ${ }^{[27]}$, 在轨运行 2 年. Xiangli 等 ${ }^{[28]}$ 研制的我国 “环境- $1 \mathrm{~A}$ ” 卫星高光谱成像仪于 2008 年发射, 至 今仍在轨稳定运行.

\section{5 时空调制 Fourier 变换成像光谱技术}

为了充分发挥 Fourier 变换光谱技术的高通量优点, 同时兼顾紧凑性和稳定性, Xiangli ${ }^{[29]}$ 提出一 种大孔径静态成像光谱技术方案, 原理如图 7 所示, 由前置镜、准直镜、横向剪切干涉仪、Fourier 镜和 探测器组成. 其中, 横向剪切干涉仪将一次像面连同准直镜一起横向剪切, 经 Fourier 镜成像在探测器 处发生干涉, 所形成的干涉图像看起来是在几何图像上叠加了坚直的干涉条纹. 干涉图像在整个像面 空间被相干调制, 这类似于空间调制, 同时, 目标每一点的完整干涉图需要沿垂直干涉条纹方向扫描整 个视场后才能获得, 是时间的函数, 因此, 该方案称为时空调制 Fourier 变换成像光谱技术 (TSMFTIS), 其主要特点是既没有狭缝也没有动镜.

由于没有入射狭缝, 时空调制 Fourier 变换成像光谱仪的辐通量与照相机相当, 远高于一般成像 光谱仪. 通常, 其光瞳面积比棱镜色散、光栅衍射和空间调制干涉成像光谱仪大两个数量级, 因此, 其 辐通量也高两个数量级.

时空调制 Fourier 变换成像光谱仪的干涉图公式与式 (16) 相同, 但其采集方式完全不同, 如图 8 所示. 在干涉图像中, 光程差与像面空间坐标的关系是固定的且在同一列上相等, 采集干涉图时, 目标 沿垂直干涉条纹方向扫过整个视场. 考察设定的某一列, 在 $t_{1}$ 时刻, 该列位于横坐标 $x_{1}$, 干涉强度向 量为 $\boldsymbol{I}\left(x_{1}\right)$, 在 $t_{2}$ 时刻, 该列位于横坐标 $x_{2}$, 干涉强度向量为 $\boldsymbol{I}\left(x_{2}\right)$, 以此类推, 在 $t_{n}$ 时刻, 该列位于 横坐标 $x_{n}$, 干涉强度向量为 $\boldsymbol{I}\left(x_{n}\right)$. 将不同时刻、位于不同干涉图像中不同位置的 $\boldsymbol{I}\left(x_{j}\right)$ 提取出来, 重 

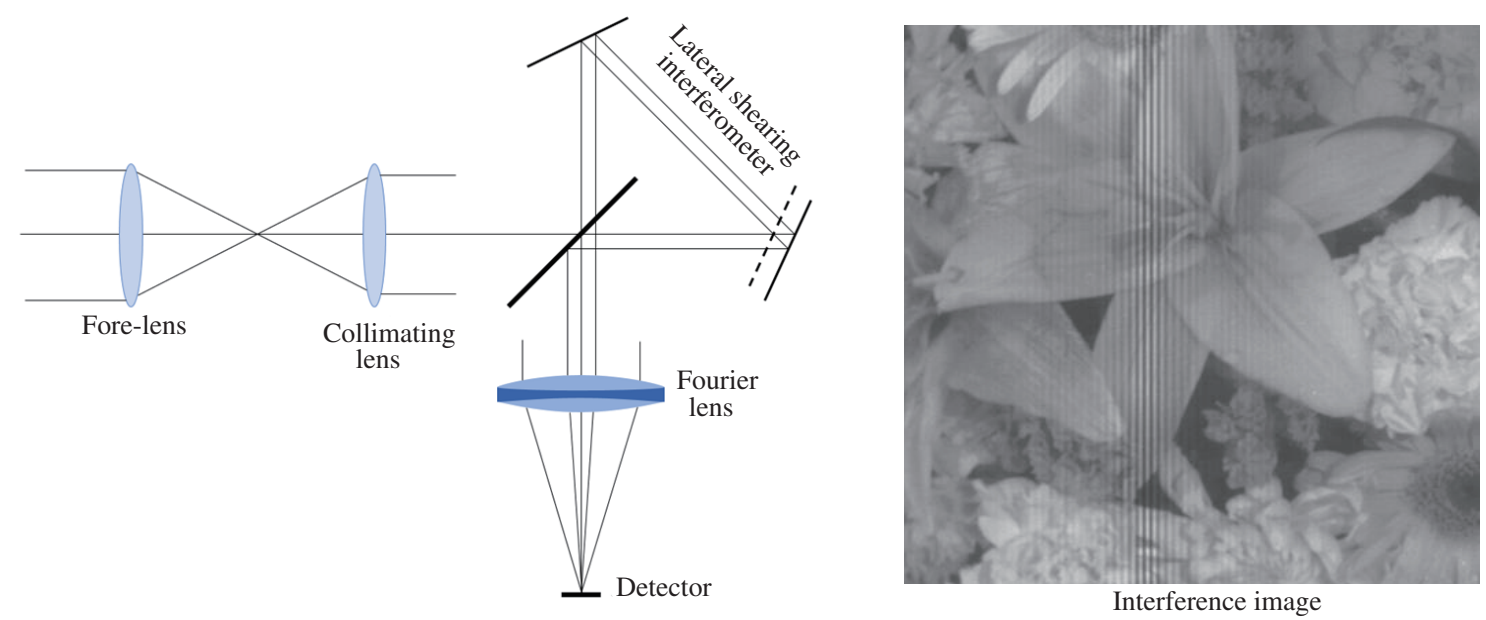

Interference image

图 7 (网络版彩图) TSMFTIS 原理示意图及干涉图像

Figure 7 (Color online) Principle of TSMFTIS and the interference image

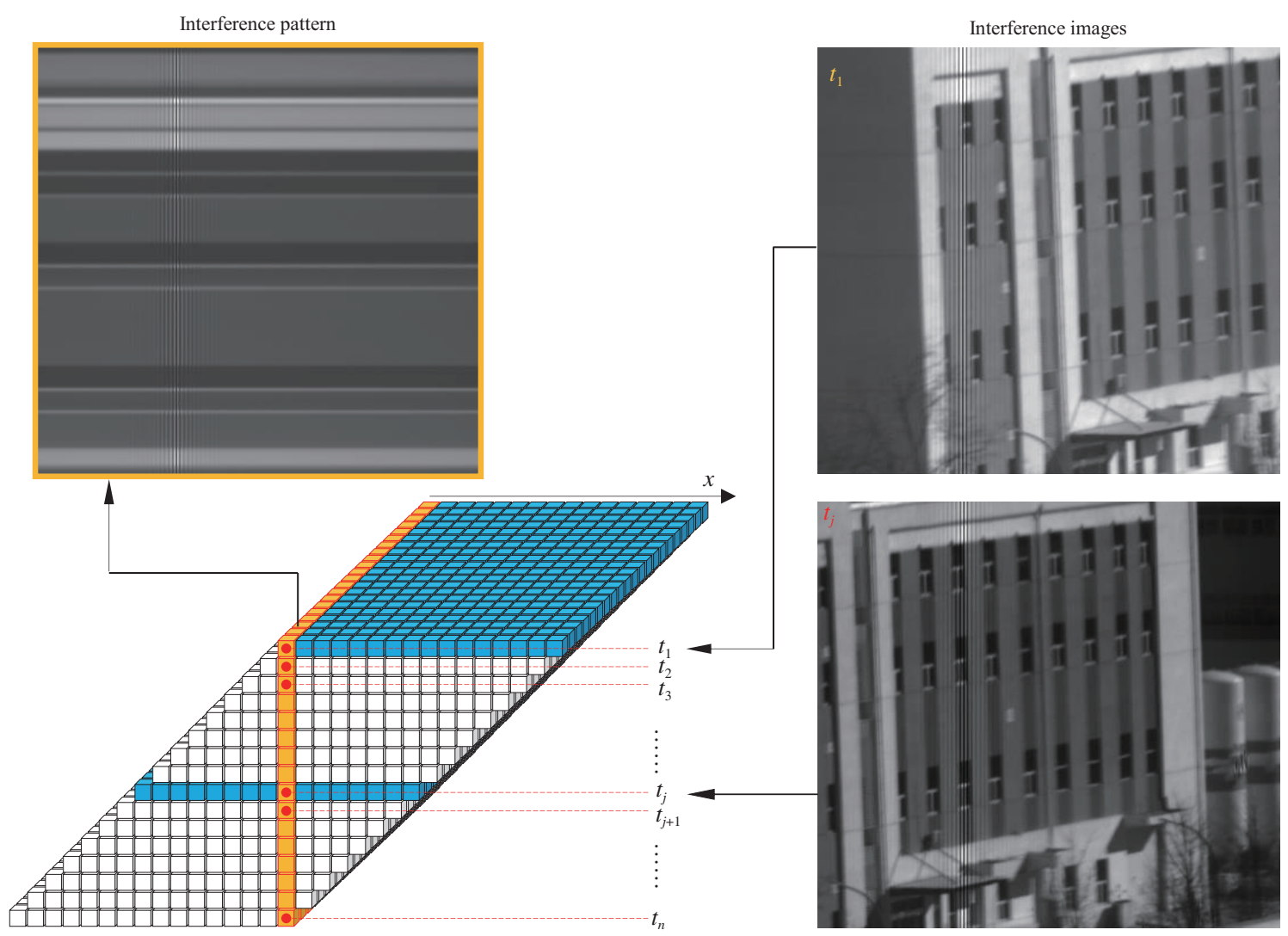

图 8 (网络版彩图) TSMFTIS 干涉数据获取示意图

Figure 8 (Color online) Interferogram extraction procedure of TSMFTIS

新排列就构成了该列的干涉图案. 可以看到, 该干涉图案与图 5 所示的空间调制 Fourier 变换成像光 谱仪某一时刻全狭缝干涉图案在形式上是相同的. 


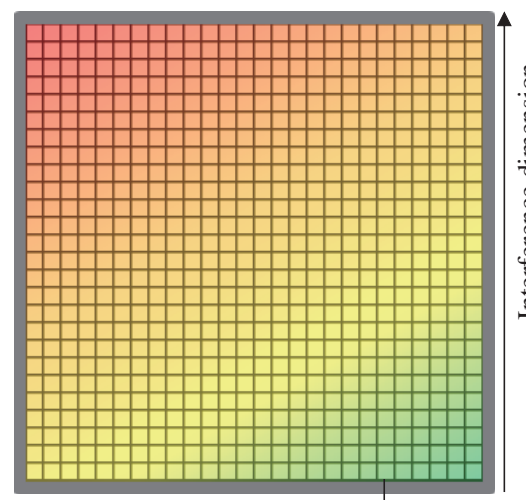

Detector

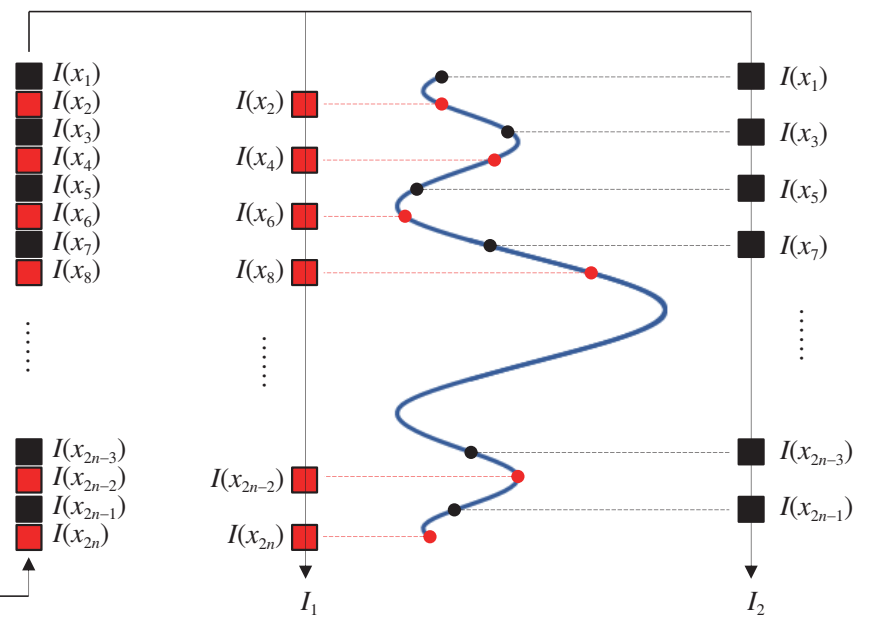

图 9 (网络版彩图) 并行采样方法示意图

Figure 9 (Color online) Schematic diagram of parallel sampling method

干涉图像某列的干涉强度向量既可以逐列采集, 也可以跨过 $k$ 列采集, 根据 Nyquist 采样定理, 只 需满足

$$
k s \leqslant \frac{f^{\prime}}{2 \zeta \nu} .
$$

图 9 描述了 $k=2$ 时干涉强度的采集过程. 在满足式 (22) 条件下, 奇数列和偶数列都满足采样定 理, 通过隔列曝光采集探测器的所有数据, 实际上是在二分之一帧频情况下, 获取 2 列目标的干涉图, 该技术称为并行采样 [30]. 当然, $k$ 值可以大于 2 , 此时探测器的帧频降低 $k$ 倍.

高通量和低帧频的特点, 使时空调制 Fourier 变换成像光谱仪成为实现高空间分辨率高光谱成像 探测的一种强有力的新手段. 以航天遥感为例, 设卫星相对地速为 $6800 \mathrm{~m} / \mathrm{s}$, 空间分辨率为 $2.5 \mathrm{~m}$, 此 时, 大面阵探测器帧频在逐列采样时高达 $2720 \mathrm{fps}$, 采用并行采样技术, 取 $k=4$ 可将探测器帧频降低 到 $680 \mathrm{fps}$; 另一方面, 以曝光像移 0.5 像元计算, 单次曝光探测器积分时间约为 $0.179 \mathrm{~ms}$, 光学系统采 用适中的 $F$ 数, 探测器在零光程差位置输出信号的电子数即可达饱和电子数的 $75 \%$. 该案例的详细设 计参数如表 2 所示, 基于该参数的星载成像光谱系统无需运动补偿, 以连续推扫的工作模式, 仍能满 足探测器帧频和系统灵敏度要求.

时空调制 Fourier 变换成像光谱仪虽然在辐通量、采样帧频、稳定性等方面优势突出, 但其干涉 图案的精准采集依赖于目标与仪器相对运动的方向性和速度均匀性, 这对干涉图案的提取和处理算法 提出了新要求.

1993 年, 美国 Horton 等 ${ }^{[31]}$ 研制了高效能成像 Fourier 变换光谱仪 HEIFTS, 开展了原理验证试 验. 2005 年, 意大利 Barducci 等 ${ }^{[32]}$ 研制了星载静态成像干涉仪 ALISEO, 尚未发射. Xiangli 等研制 的我国 “遥感 - 14” 卫星大孔径静态成像光谱仪 LASIS, 于 2012 年、 2014 年相继发射, 是国际上迄今 唯一开展航天遥感应用的时空调制 Fourier 变换成像光谱仪.

\section{6 小结}

Fourier 变换成像光谱技术在科技进步和应用需求的双重驱动下迅猛发展, 本文按照 3 种调制方 式对 FTIS 的基本原理、典型方案、关键技术和主要应用进行了概要介绍. 事实上, FTIS 涉及的理论、 


\section{表 2 高空间分辨率高光谱成像系统设计参数}

Table 2 Design characteristics of the high spatial resolution hyper-spectral imager

\begin{tabular}{cc}
\hline Instrument parameter & Instrument characteristic \\
\hline Orbit altitude & $500 \mathrm{~km}$ \\
Imaging mode & Continuous pushbroom \\
Ground sampling distance & $2.5 \mathrm{~m}$ \\
Spectral coverage & $400-1000 \mathrm{~nm}$ \\
Number of bands & 64 \\
Parallel sampling times & 4 \\
Maximum frame rate of detector & $700 \mathrm{fps}$ \\
Pixel size & $16 \mu \mathrm{m}$ \\
Quantum efficiency & $0.81 @ 645 \mathrm{~nm}$ \\
Full well capacity & $200000 \mathrm{e}^{-}$ \\
F\# & 5 \\
Integration time & $0.179 \mathrm{~ms}$ \\
Solar elevation angle & $70^{\circ}$ \\
Albedo & 0.3 \\
Output electrons of zero OPD & $150156 \mathrm{e}^{-}$ \\
SNR of the interferogram at the center burst & 500 \\
\hline
\end{tabular}

技术和应用非常丰富, 本文未能全面覆盖. 比如空间外差式 FTIS ${ }^{[33,34]}$, 能够在较窄的谱段范围内, 以 较少的干涉图采样点数, 实现极高的光谱分辨率.

展望未来, Fourier 变换光谱以及相关干涉技术, 仍将活跃在科技发展和社会进步的最前沿. 时间 调制 FTIS 的高灵敏度、高光谱分辨率和高信噪比等优点, 将为精准气象预报、环境监测、污染检测 等提供有力支撑, 也将成为探索宇宙的重器 ${ }^{[35]}$. 时空调制 FTIS 的高灵敏度、高稳定性等优点, 使其 能够在航天遥感等应用领域不断突破, 成为高效率、高精度综合信息感知的利器. 各种干涉仪一直是 精密测量、光谱分析、量子科技等诸多领域不可或缺的基本工具, Michelson 干涉仪历久弥新, 已经在 地面探测到引力波 ${ }^{[36]}$, 未来还将在空间引力波探测等极大尺度超高精度测量方面发挥更大作用.

\section{参考文献}

1 Newton I. Opticks: Or A Treatise of the Reflexions, Refractions, Inflexions and Colours of Light. London: Royal Society, 1704

2 Kirchhoff G, Bunsen R. Chemical analysis by observation of spectra. Ann der Physik und der Chem (Poggendorff), 1860, 110: 598-625

3 Michelson A A. Studies in Optics. New York: Dover Publications, 1995

4 Michelson A A, Morley E W. On the relative motion of the earth and the luminiferous ether. Am J Sci, 1887, 34: 333-345

5 Rayleigh L. On the interference bands of approximately homogeneous light: in a letter to Prof. A. Michelson. Phil Mag, 1892, 34: 407-411

6 Cooley J W, Tukey J W. An algorithm for the machine calculation of complex Fourier series. Math Comp, 1965, 19: $297-297$

7 Jacquinot P, Dufour C. Optical conditions in the use of photo-electric cells in spectrographs and interferometers. J Rech CNRS, 1948, 6: 91-103 
8 Fellgett P. Theory of multiplex interferometric spectrometry. J Phys Radium, 1958, 19: 187-191

9 Goetz A F H, Vane G, Solomon J E, et al. Imaging spectrometry for earth remote sensing. Science, 1985, 228: $1147-1153$

10 Norton R H, Beer R. New apodizing functions for Fourier spectrometry. J Opt Soc Am, 1976, 66: 259-264

11 Mertz L. Auxiliary computation for Fourier spectrometry. Infrared Phys, 1967, 7: 17-23

12 Forman M L, Steel W H, Vanasse G A. Correction of asymmetric interferograms obtained in Fourier spectroscopy*. J Opt Soc Am, 1966, 56: 59-63

13 Schott J R. Remote Sensing: The Image Chain Approach. 2nd ed. Oxford: Oxford University Press, 2007

14 Fiete R D. Comparison of SNR image quality metrics for remote sensing systems. Opt Eng, 2001, 40: 574-585

15 Kelly M W, Mooney D L. A method for correcting Fourier transform spectrometer (FTS) dynamic alignment errors. Proc SPIE, 2004, 5425: 443-455

16 Rippel H, Jaacks R. Performance data of the double pendulum interferometer. Mikrochim Acta, 1988, 95: 303-306

17 Griffiths P R, Hirsche B L, Manning C J. Ultra-rapid-scanning Fourier transform infrared spectrometry. Vibal Spectr, 1999, 19: $165-176$

18 Kauppinen J, Heinonen J, Kauppinen I. Interferometers based on the rotational motion. Appl Spectr Rev, 2004, 39: 99-130

19 Tanii J, Machida T, Ayada H, et al. Ocean color and temperature scanner for ADEOS. Proc SPIE, 1991, 1490: 200-206

20 Hua J W, Mao J H. Geostationary interferometric-type infrared sounder (GIIRS) on FengYun No.4 metrological satellite. Science, 2018, 70: 24-29 [华建文, 毛建华. “风云四号” 气象卫星大气垂直探测仪. 科学, 2018, 70: 24-29]

21 Bernath P. Atmospheric chemistry experiment (ACE): analytical chemistry from orbit. Trends Anal Chem, 2006, 25: $647-654$

22 Hamazaki T, Kaneko Y, Kuze A, et al. Fourier transform spectrometer for greenhouse gases observing satellite. Proc SPIE, 2005, 5659: 73-80

23 Jiang C, Tao D X, He H Y. Digital modeling and simulation of AIUS. Spacecr Recovery Remote Sens, 2018, 39: 94-103 [江澄, 陶东兴, 何红艳. 大气环境红外甚高光谱分辨率探测仪数字建模与仿真. 航天返回与遥感, 2018, 39: 94-103]

24 Lucey P G, Horton K A, Williams T J, et al. SMIFTS: a cryogenically-cooled spatially modulated imaging infrared interferometer spectrometer. Proc SPIE, 1993, 1937: 130-141

25 Xiangli B, Zhao B C, Xue M Q. Spatially modulated imaging interferometry. Acta Opt Sin, 1998, 18: 18-22 [相里斌, 赵葆常, 薛鸣球. 空间调制干涉成像光谱技术. 光学学报, 1998, 18: 18-22]

26 Xiangli B, Yuan Y, Lv Q B. Spectral transfer function of the Fourier transform spectral imager. Acta Phys Sin, 2009, 58: 5399-5405 [相里斌, 袁艳, 吕群波. 傅里叶变换光谱成像仪光谱传递函数研究. 物理学报, 2009, 58: 5399-5405]

27 Yarbrough S, Caudill T, Kouba M E, et al. MightySat II.1 hyperspectral imager: summary of on-orbit performance. Proc SPIE, 2002, 4480: 186-197

28 Xiangli B, Wang Z H, Liu X B, et al. Hyperspectral imager of the environment and disaster monitoring small satellite. Remote Sens Technol Appl, 2009, 24: 257-262 [相里斌, 王忠厚, 刘学斌, 等. “环境与灾害监测预报小卫星” 高光谱 成像仪. 遥感技术与应用, 2009, 24: 257-262]

29 Xiangli B. Interferometric imaging spectrometry. Post-Doctoral Final Technical Report. Xi'an: Xi'an Institute of Optical and Precision Mechanics, Chinese Academy of Sciences, 1995-1997 [相里斌. 干涉成像光谱技术研究. 博士后 研究工作总结报告. 西安: 中国科学院西安光学精密机械研究所, 1995-1997]

30 Xiangli B, Huang M, Liu X B, et al. Parallel Sampling Method for LASIS. China Patent, ZL 200710017721.3 [相里 斌, 黄旻, 刘学斌, 等. 大孔径静态干涉成像光谱仪并行采样方法. 中国发明专利, ZL 200710017721.3]

31 Horton R F, Conger C A, Pellegrino L S. High etendue imaging Fourier transform spectrometer: initial results. Proc SPIE, 1997, 3118: 380-390

32 Barducci A, Castagnoli F, Marcoionni P, et al. The ALISEO instrument: further improvements of calibration methods and assessment of interferometer response. Proc SPIE, 2005, 5978: 461-470

33 Harlander J M, Roesler F L, Cardon J G, et al. Shimmer: a spatial heterodyne spectrometer for remote sensing of earth's middle atmosphere. Appl Opt, 2002, 41: 1343-1352

34 Xiangli B, Cai Q S, Du S S. Large aperture spatial heterodyne imaging spectrometer: principle and experimental results. Opt Commun, 2015, 357: 148-155

35 Hansen S M. Spectral line position calibration for the SPIRIT III Fourier transform spectrometer. Opt Eng, 1997, 36: 
2987-2991

36 Althouse W E, Hand S D, Jones L K, et al. Precision alignment of the LIGO $4 \mathrm{~km}$ arms using the dual-frequency differential global positioning system. Rev Sci Instrum, 2001, 72: 3086-3094

\title{
Fourier transform imaging spectroscopy
}

\author{
Bin XIANGLI*, Qunbo LV, Qisheng CAI, Yu FANG, Jinsong ZHOU \& Min HUANG \\ Key Laboratory of Computational Optical Imaging Technology, Aerospace Information Research Institute, Chinese \\ Academy of Sciences, Beijing 100094, China \\ * Corresponding author. E-mail: xiangli@cashq.ac.cn
}

\begin{abstract}
Imaging spectrometry can obtain both geometric image and spectral radiation of targets. Prisms, gratings, and interferometers are often used for light decomposition. Fourier transform imaging spectroscopy (FTIS) is based on light interference to obtain interferograms of the target and reconstruct the spectra using the Fourier transform method. FTIS has numerous advantages, including high throughput and spectral resolution and multiplex. It is a hotspot in the field of imaging spectroscopy and has been established in three types, namely, temporally modulated, spatially modulated, and temporally-spatially modulated. FTIS has been widely used in various fields, including industry, agriculture, scientific research, biomedicine, atmospheric detection, environmental monitoring, and resource investigation. In this paper, the basic principle of FTIS based on the Michelson interferometer is briefly introduced. Typical techniques for different modulation types and remote sensing applications are also presented.
\end{abstract}

Keywords Fourier transform, imaging spectroscopy, throughput, multiplex, modulation depth, parallel sampling

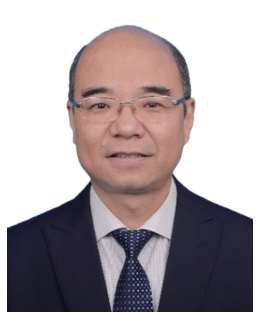

Bin XIANGLI was born in 1967. He received his B.S. degree from the University of Science and Technology of China in 1990 and Ph.D. degree from Xi'an Institute of Optics and Precision Mechanics, Chinese Academy of Sciences (CAS) in 1995. At present, he is the vice president of CAS. He is an academician of CAS and chairman of the Key Laboratory of Computational Optical Imaging Technology of CAS. His primary research interests include optical engineering technology, particularly the fields of aerospace and aviation hyperspectral imaging remote sensing.

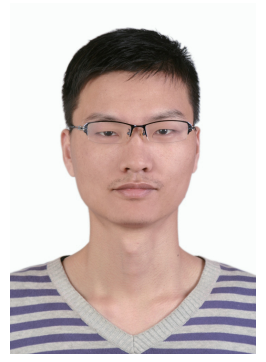

Qisheng CAI was born in 1988. He received his B.S. degree from University of Science and Technology of China in 2011 and Ph.D. degree from the University of Science and Technology of China in 2016. He works at the Key Laboratory of Computational Optical Imaging Technology, Aerospace Information Research Institute, Chinese Academy of Sciences (CAS). His primary research interests include the study of hyperspectral imaging technology.

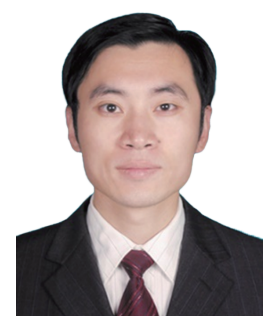

Qunbo LV was born in 1979. He received his B.S. degree from Xidian University in 2001 and Ph.D. degree from Xi'an Institute of Optics and Precision Mechanics, Chinese Academy of Sciences (CAS) in 2007. He works at the Key Laboratory of Computational Optical Imaging Technology, Aerospace Information Research Institute, CAS. His primary research fields include optical imaging technology and image process-

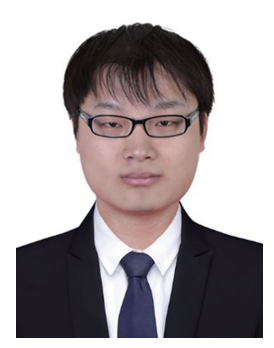

Yu FANG was born in 1987. He received his B.S. degree from University of Science and Technology of China in 2008 and Ph.D. degree from Xi'an Institute of Optics and Precision Mechanics, Chinese Academy of Sciences (CAS) in 2013. At present, he works at the Key Laboratory of Computational Optical Imaging Technology, Aerospace Information Research Institute, CAS. His primary research interests include spectral imaging technology research and optical system design. 